\section{Progression of mild cognitive impairment}

\section{to dementia: a challenge to current thinking*}

ANJA BUSSE, MATTHIAS C. ANGERMEYER and STEFFI G. RIEDEL-HELLER
Recognition of mild cognitive impairment as a transition phase between healthy ageing and dementia is important in the investigation of treatments aimed at secondary prevention of dementia. People with mild cognitive impairment progressed to dementia in several studies at very different rates, with an average conversion rate of $10 \%$ per year (Petersen et al, 2001; DeCarli, 2003; Bruscoli \& Lovestone, 2004; Petersen, 2004a; Panza et al, 2005), suggesting a linear progression of conversion to dementia over time. Petersen (2003) reports that after approximately 6 years, $80 \%$ of the mild cognitive impairment cohort has progressed to dementia. The final proportions may even extend to $80-90 \%$. Most of the current knowledge stems from clinical samples. Using a representative general population sample, this study examined the time-dependent evolution of mild cognitive impairment to dementia over an observation period of 6 years. We examined what proportion of people with mild cognitive impairment at each follow-up assessment developed dementia, and how many people with mild cognitive impairment died before meeting diagnostic criteria of clinical dementia.

\section{METHOD}

dependent evolution from mild cognitive impairment to dementia and to establish age-specific conversion rates during lifetime.

\section{Declaration of interest None.} Funding detailed in Acknowledgements.

*Results of the Leipzig Longitudinal Study of the Aged (LEILA75+) the Leipzig Longitudinal Study of the Aged has been described in detail elsewhere (Riedel-Heller et al, 2001).

Of the overall sample of 1692 persons, $242(14.3 \%)$ declined to participate, 57 (3.4\%) had died and 15 (0.9\%) were not traceable. Information on 113 members of the study sample $(6.7 \%)$ who were shielded by their relatives was obtained solely by proxy interviews. Clinical interviews incorporating neuropsychological assessment were conducted with 1265 (74.8\%) people who did not differ significantly from the remainder of the sample in terms of age $(U=263553, P=0.455)$, gender $\left(\chi^{2}=0.391\right.$, d.f. $=1, \quad P=0.532) \quad$ or marital status $\left(\chi^{2}=5.027\right.$, d.f. $\left.=3, \quad P=0.170\right)$. Of these 1265 people, $220(17.4 \%)$ had dementia according to DSM-IV criteria (American Psychiatric Association, 1994). This analysis was based on the remaining 1045 without dementia.

\section{Instruments}

The main instrument used was the Structured Interview for Diagnosis of Dementia of Alzheimer-type, Multi-infarct Dementia and Dementia of other Aetiology according to ICD-10 (World Health Organization, 1992) and DSM-IV (SIDAM; Zaudig et $a l, 1991)$. The SIDAM consists of a neuropsychological test battery including the Mini-Mental State Examination (MMSE; Folstein et al, 1975), a section for clinical judgement and third-party information on psychosocial impairment. The neuropsychological test battery of the SIDAM covers six areas of neuropsychological functioning:

(a) orientation - assessment of orientation for time and place;

(b) memory - measured by delayed verbal recall of a word list and a fictitious name and address, and delayed visual reproduction, questions on biographical knowledge and on historical data unrelated to the person's life;

(c) intellectual abilities - assessed by items of abstract thinking (differences, explaining the meaning of idiomatic expressions) and judgement (describing pictures representing actions, and plausibility judgement);

(d) verbal abilities and calculation assessed by calculating serial sevens, spelling backwards, and digit span backwards; 
(e) constructional abilities (visuospatial) assessed by copying figures;

(f) aphasia and apraxia - assessed by naming objects, reading and obeying a sentence, writing a sentence, and performing a three-stage command.

For each cognitive domain, age-specific and education-specific norms were used in the evaluation of impairment in cognitive function. The norms were developed from the baseline population (participants without dementia) from which the study sample was recruited (Busse et al, 2002).

Data on socio-demographic variables and possible risk factors for dementia and mild cognitive impairment were collected. A series of validated scales examining the capacity to perform a wide range of activities of daily living, such as use of telephone, feeding, dressing and personal hygiene, were completed. Complaints of subjective memory impairment were assessed before cognitive testing by asking participants if they had any problems with their memory (answer 'yes' or 'no'). Depressive symptoms were assessed by means of the Center for Epidemiological Studies Depression scale (CES-D; Radloff, 1977) and the Structured Clinical Interview for DSM-III-R (SCID; Spitzer et al, 1987).

\section{Data collection}

Structured clinical interviews were conducted by trained psychologists and physicians during visits to the participants' homes. In addition, structured third-party interviews were conducted to obtain information on cognitive and psychosocial functioning and subjective memory impairment. Baseline interviews were conducted between January 1997 and June 1998. Study participants were requested to take part in four follow-up assessments, which were conducted 1.5 years, 3 years, 4.5 years and 6 years after the baseline assessment.

If it was not possible to administer the SIDAM at follow-up (e.g. owing to death or severe weakness, or because relatives refused participation on behalf of the elderly person in their care), we offered the option of a fully structured proxy interview. This included the Clinical Dementia Rating scale (CDR; Hughes et al, 1982) for assessment of cognitive functioning.

Study participants were followed until death or onset of dementia or for a maximum of 6 years if the SIDAM could be administered at each follow-up assessment. As long as the SIDAM could be administered to apply diagnostic criteria of mild cognitive impairment and dementia, participants remained in the study. If participants refused further participation, or if there was not sufficient information to apply diagnostic criteria of mild cognitive impairment or dementia, they were excluded from the study. The statistical analysis for these was based on the diagnosis established at the last follow-up visit at which the participant had undergone cognitive testing. In case of death or onset of dementia, the diagnostic criteria of dementia were applied based on CDR data or based on the SIDAM.

\section{Definition of mild cognitive impairment}

Consensus conferences of physicians and psychologists were held for each participant. The clinical diagnosis of dementia was made according to DSM-IV criteria.

According to Petersen (2004b), four subtypes of mild cognitive impairment were examined:

(a) amnesic mild cognitive impairment, single domain - isolated memory impairment of more than 1.0 s.d. compared with the age- and education-specific norms, and no difficulty in any other area of cognitive functioning;

(b) amnesic mild cognitive impairment, multiple domain - two or more cognitive domains are impaired, one of which is memory impairment (impairment of more than 1.0 s.d. below the mean of the respective age- and educationmatched population);

(c) non-amnesic mild cognitive impairment, single domain - impairment in a single domain other than memory of more than 1.0 s.d.;

(d) non-amnesia mild cognitive impairment, multiple domain - impairments in two or more domains of more than 1.0 s.d. but no memory impairment.

In the following analysis, mild cognitive impairment was diagnosed if diagnostic criteria of one of the four subtypes was fulfilled. All four subtypes of mild cognitive impairment also had to meet the following criteria:

(a) the presence of a complaint about memory - participants or informants (or both) reporting memory impairment;

(b) intact ability to perform activities of daily living - forgetfulness not compromising overall functional ability; impairment owing to physical disease not sufficient for exclusion;

(c) absence of dementia - assessed by DSM-IV criteria.

In addition to the cut-off of 1.0 s.d. used to define cognitive impairment (mild cognitive impairment original 1.0 s.d.), a cut-off of 1.5 s.d. was also used to capture only the more severely affected individuals (mild cognitive impairment original 1.5 s.d.). Furthermore, we introduced slightly modified criteria by excluding the first criterion (the presence of a complaint) from the necessary diagnostic criteria on memory (mild cognitive impairment 1.0 modified, mild cognitive impairment 1.5 modified). The importance of subjective memory impairment in the prediction of dementia is subject to debate. Some authors suggest that memory complaints may not be of additional predictive value (Jorm et al, 1997), others have shown that memory complaints are an important predictor for progressive cognitive decline (Comijs et al, 2004).

People with a diagnosis of Parkinson's disease, learning disability and brain cancer, or severe weakness or severe sensory impairment leading to invalid cognitive testing were excluded from the study, whereas persons with depression or stroke were not excluded.

\section{Analysis}

In order to analyse possible non-response bias, $\chi^{2}$ analysis and the Mann-Whitney $U$-test were applied. For all analyses, an $\alpha$ level of 0.01 was used. Descriptive statistics were used for the demographic variables. Cumulative numbers of people and cumulative percentages (related to the number of participants at each follow-up) are given for each point of assessment to illustrate the time-dependent progression of mild cognitive impairment to dementia or death. Estimation of the maximum conversion rate of mild cognitive impairment to dementia during life is based on the conservative assumption that all individuals with mild cognitive impairment who are still alive after 6 years without dementia will develop dementia later.

\section{RESULTS}

\section{Baseline evaluation}

Of the 1045 people without dementia, 65 met the exclusion criteria and 980 remained for baseline examination. 
Table I Demographic characteristics of the baseline population according to different diagnostic criteria of mild cognitive impairment

\begin{tabular}{|c|c|c|c|c|c|}
\hline \multirow[t]{2}{*}{ Baseline characteristics } & \multirow{2}{*}{$\begin{array}{c}\text { Total } \\
\text { baseline } \\
\text { population }\end{array}$} & \multicolumn{4}{|c|}{ Mild cognitive impairment } \\
\hline & & $\begin{array}{c}\text { original } \\
\text { I.0 s.d. }\end{array}$ & $\begin{array}{c}\text { original } \\
\text { I.5 s.d. }\end{array}$ & $\begin{array}{c}\text { modified } \\
\text { I.0 s.d. }\end{array}$ & $\begin{array}{c}\text { modified } \\
\text { I.5 s.d. }\end{array}$ \\
\hline Number of participants, $n$ (\%) & 980 & $189(19.3)$ & 91 (9.3) & $407(41.5)$ & $169(17.2)$ \\
\hline Age, in years: mean (s.d.) & $81.5(4.8)$ & $82.2(5.0)$ & $82.1(4.8)$ & $81.6(4.9)$ & $81.8(4.8)$ \\
\hline MMSE score: mean (s.d.) & $27.0(2.1)$ & $25.4(2.0)$ & $24.8(2.1)$ & $25.8(2.1)$ & $24.9(2.0)$ \\
\hline \multicolumn{6}{|l|}{ Gender, $n(\%)$} \\
\hline Male & $246(25.1)$ & $56(29.6)$ & $27(29.7)$ & $105(25.8)$ & $44(26.0)$ \\
\hline Female & 734 (74.9) & I 33 (70.4) & $64(70.3)$ & $302(74.2)$ & $125(74.0)$ \\
\hline \multicolumn{6}{|l|}{ Level of education, $n$ (\%) } \\
\hline Low & $220(22.4)$ & $44(23.3)$ & $15(16.5)$ & $92(22.6)$ & $35(20.7)$ \\
\hline Middle & $624(63.7)$ & $116(61.4)$ & $62(68.1)$ & $252(61.9)$ & $109(64.5)$ \\
\hline High & $135(13.8)$ & $28(14.8)$ & $13(14.3)$ & $62(15.2)$ & $24(14.2)$ \\
\hline Missing & I (0.I) & I (0.5) & I (I.I) & I (0.2) & I (0.6) \\
\hline Drop-outs after baseline, $n(\%)$ & $117(11.9)$ & $25(13.2)$ & II (12.I) & $61(15.0)$ & $25(14.8)$ \\
\hline
\end{tabular}

$\mathrm{MCl}$, mild cognitive impairment; MMSE, Mini-Mental State Examination. $\mathrm{MCl}$ original I.0 s.d. $-\mathrm{MCl}$ according to Petersen (2004); $\mathrm{MCl}$ original I.5 s.d. - MCl according to Petersen (2004) with changed cut-off (I.5 s.d.) to define cognitive impairment; $\mathrm{MCl}$ modified 1.0 s.d. $-\mathrm{MCl}$ according to Petersen (2004) modified by omitting criterion on memory complaint; $\mathrm{MCl}$ modified I.5 s.d. - MCl according to Petersen (2004) with changed cut-off (I.5 s.d.) to define cognitive impairment and modified by omitting criterion on memory complaint.

Baseline characteristics of the total study sample are given in Table 1 . The study population had a mean age of 81.5 years (s.d.=4.8). Three-quarters of the sample were women. The mean MMSE score was 27 points.

(a)

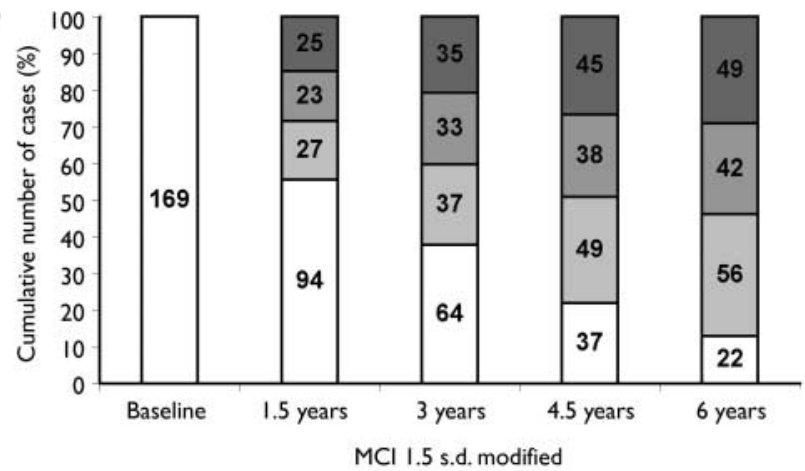

(c)

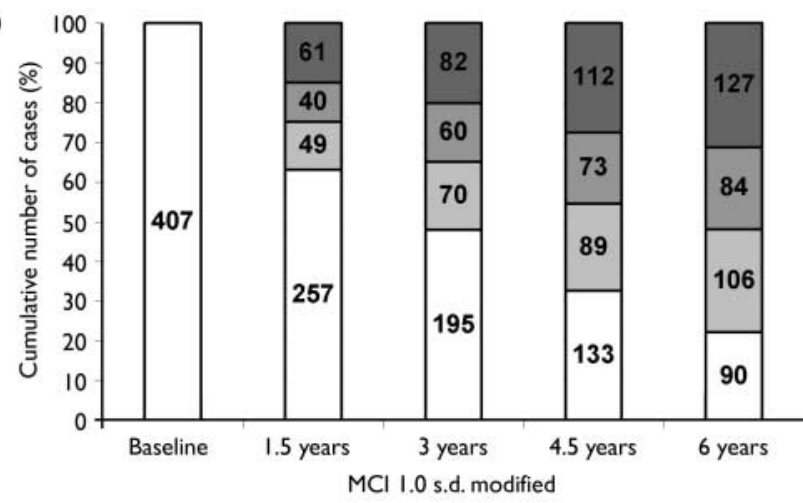

Table 1 also reports baseline characteristics according to the four different diagnostic criteria of mild cognitive impairment. The mean age was 82 years (to the nearest year) in all four groups; the mean MMSE score was 25-26 points. The original criteria of mild cognitive impairment applied to $19 \%$ of the study participants if a cut-off of 1.0 s.d. was chosen. If the criterion of subjective memory impairment was excluded (modified criteria), the baseline prevalence increased to $42 \%$ (cutoff of 1.0 s.d.). A similar pattern was observed if a cut-off level of 1.5 s.d. was chosen: the prevalence of mild cognitive impairment almost doubles if modified criteria are applied $(17 \% v .9 \%)$. If a cutoff level of 1.5 s.d. was chosen as opposed to a level of 1 s.d., the prevalence of mild cognitive impairment (original or modified criteria) decreased by about $50 \%$.

\section{Examination at follow-up}

After baseline examination, 117 (11.9\%) participants had to be excluded from analysis because they refused further participation, or there was not sufficient information to apply diagnostic criteria of mild cognitive impairment or dementia. These 117 participants did not differ significantly from the remainder of the sample $(n=863$, $88.1 \%)$ in age ( $U=46491, P=0.164)$, gender $\left(\chi^{2}=5.551\right.$, d.f. $\left.=1, \quad P=0.058\right)$, education $\left(\chi^{2}=3.268\right.$, d.f. $\left.=2, P=0.195\right)$ or complaints of impaired memory expressed by the individual or significant others at the baseline assessment $\left(\chi^{2}=4.781\right.$, d.f. $\left.=3, P=0.189\right)$.
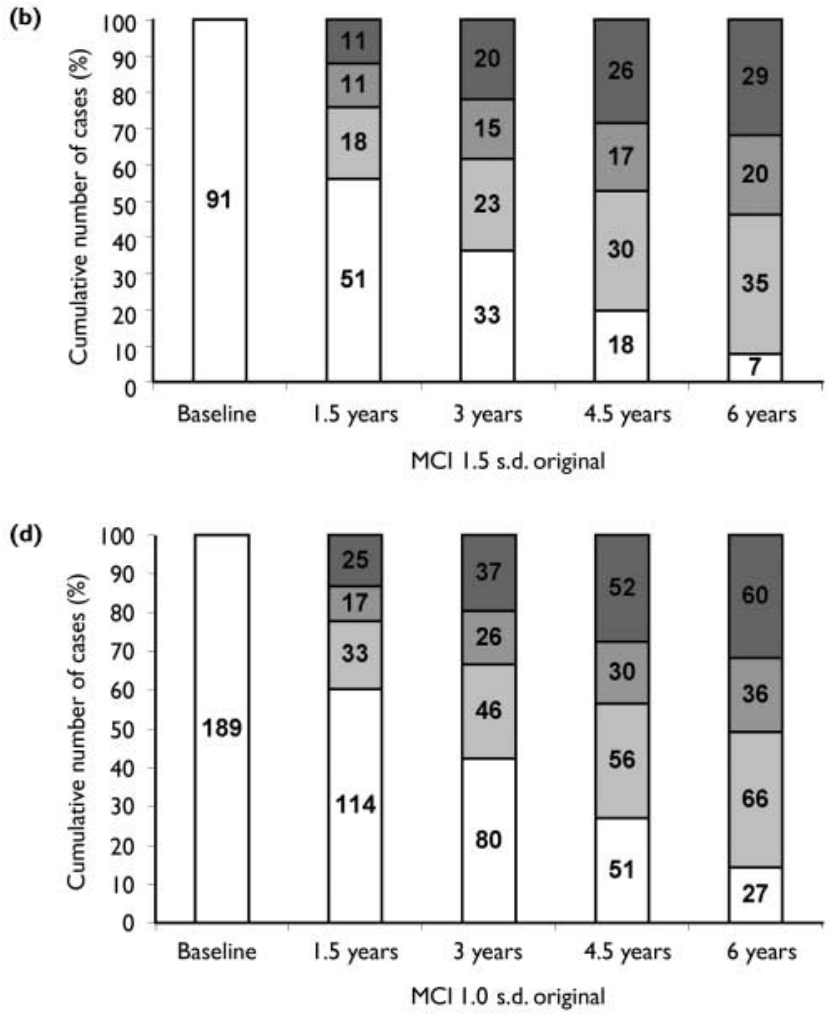

Fig. I Number of study participants at baseline and at follow-up with mild cognitive impairment ( $\mathrm{MCl}$; Petersen, 2004) according to different diagnostic criteria: (a) $\mathrm{MCl}$ I.5 s.d. modified; (b) MCI I.5 s.d. original; (c) MCl I.0 s.d. modified; (d) MCl I.0 s.d. original. $\square$, MCl; $\square$, dementia; $\square$, deceased, no dementia; $\square$, drop-out. 

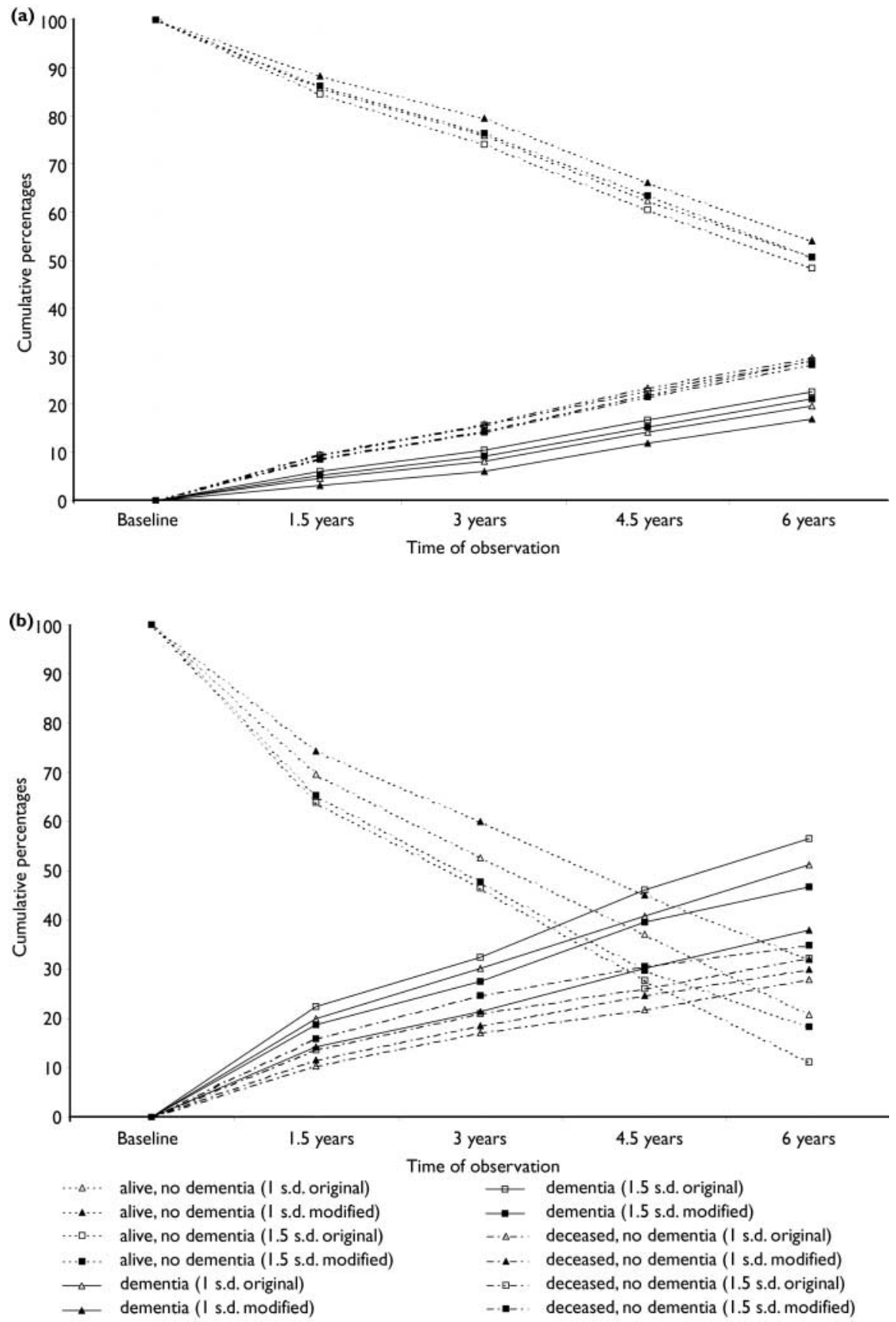

Fig. 2 Cumulative percentages for different outcomes of people with (a) non-mild and (b) mild cognitive impairment at baseline related to the total number of participants who remained in the study at each follow-up assessment.

However, drop-outs had a significantly lower MMSE score at baseline $(U=41597$, $P=0.002)$. Drop-out rates reported in Table 1 suggest that drop-out was slightly higher in the groups with mild cognitive impairment than in the total study population. However, comparing drop-outs in the mild cognitive impairment group with drop-outs in the corresponding non-mild cognitive impairment group for each of
$(22.6 \%)$ died without a diagnosis of dementia during the entire observation period.

\section{Outcome of mild cognitive impairment at each follow-up assessment over 6 years}

Figure 1 shows the cumulative number for each follow-up assessment of study participants who developed dementia, died without a diagnosis of dementia, were alive without a dementia diagnosis or refused further participation.

Figure 2 shows the cumulative percentages of participants who developed dementia, died without a diagnosis of dementia or were alive without dementia diagnosis, related to the total number of participants who remained in the study at each follow-up assessment.

After 6 years of observation, about $50 \%$ of the participants who did not meet diagnostic criteria of mild cognitive impairment at baseline (non-mild cognitive impairment), and underwent further followup assessment, were still alive without a diagnosis of dementia; about $30 \%$ died; and about $20 \%$ developed dementia. At each follow-up assessment, the proportion of participants who died without ever having a diagnosis of dementia was higher than the proportion of participants who developed dementia (Fig. 2a).

Participants who fulfilled diagnostic criteria of mild cognitive impairment at baseline, and underwent further follow-up assessment, had different outcomes. At each follow-up assessment, the proportion of participants who developed dementia was higher than the proportion who died without ever having a diagnosis of dementia. Irrespective of which diagnostic cutoff for mild cognitive impairment was applied (modified or original criteria, 1.0 or 1.5 s.d.), the proportion of participants with mild cognitive impairment who developed dementia was highest during the first 18 months of observation (between $14 \%$ and $23 \%$ ). Of participants who met modified criteria for mild cognitive impairment (1.0 s.d.), 14\% developed dementia within the first 18 months of observation, but at further follow-ups only 7-9\% did so. Participants who fulfilled the other three diagnostic concepts of mild cognitive impairment had a progression rate to dementia of about $20 \%$ within the first 18 months of observation, which reduced to $10 \%$ at further follow-up. After 6 years of observation, 
only about $20 \%$ of participants with mild cognitive impairment at baseline were still alive without a diagnosis of dementia, about $30 \%$ died without dementia, and about $50 \%$ developed dementia (Fig. $2 b$ ).

\section{Estimation of the maximum conversion rate during life}

If we tried to estimate the maximum conversion rate to dementia in participants with mild cognitive impairment, we would assume it to be about $65 \%$. According to the mild cognitive impairment modified criteria (1.5 s.d.), only 22 participants were still alive without a diagnosis of dementia after 6 years (Fig. 1). If all 22 participants developed dementia at follow-up, 78 $(56+22)$ people $(65 \%)$ would have developed dementia and 42 participants $(35 \%)$ would have died without a diagnosis of dementia. Similarly, according to the mild cognitive impairment original criteria (1.5 s.d.), after 6 years only 7 participants were still alive without a diagnosis of dementia. If all 7 participants developed dementia at follow-up, $42(35+7)$ people $(68 \%)$ would have developed dementia and 20 participants $(32 \%)$ would have died without a diagnosis of dementia. Since some of the 22 (mild cognitive impairment modified criteria, 1.5 s.d.) or 7 (mild cognitive impairment original criteria, 1.5 s.d.) would have died without a diagnosis of dementia, we could say that a maximum of $60-65 \%$ of people with mild cognitive impairment, who were 75 years or older, would develop clinical dementia.

\section{DISCUSSION}

Undoubtedly, people with mild cognitive impairment are at increased risk of developing dementia. However, our results challenge the assumption of a linear progression to dementia over time. According to our results, conversion rates to dementia in people with mild cognitive impairment are highest (about 20\%) during the first interval of observation, which was 18 months in our study. At further follow-up, conversion rates to dementia reduce to about $10 \%$ at each further point of assessment. This pattern emerges for each of the four different cut-off levels for mild cognitive impairment investigated. A similar pattern was found in two other population-based studies (Johansson et al, 1992; Palmer et $a l, 2002,2003)$. The results of these studies suggest that progression from mild

ANJA BUSSE, DPsych, MATTHIAS C. ANGERMEYER, MD, STEFFI G. RIEDEL-HELLER, MD, MPH, Leipzig Longitudinal Study of the Aged LEILA75+, Department of Psychiatry, University of Leipzig, Germany

Correspondence: Professor Steffi G. Riedel-Heller, MPH, Department of Psychiatry, University of Leipzig, Johannisallee 20, 04317 Leipzig, Germany. Tel.: +49 34I 9724 530; fax: +49 341 9724539. Email: ries@medizin.uni-leipzig.de

(First received 29 June 2005, final revision 20 March 2006, accepted 2 June 2006)

cognitive impairment to dementia is timedependent, occurring primarily within the initial 2-3 years of observation. Further studies are needed, to examine this timedependent evolution. To our knowledge, there are no such previous clinical studies. It is premature to suggest an annual conversion rate of mild cognitive impairment to dementia of $10-15 \%$, because there seems to be a higher conversion rate during the first 2-3 years of observation as compared with later assessment points.

Furthermore, our results suggest that people with mild cognitive impairment, who are aged 75 years or older, develop clinical dementia during their lifetime at a rate of approximately $60-65 \%$. In contrast, Petersen (2003) reports that after approximately 6 years $80 \%$ of the mild cognitive impairment cohort has progressed to dementia; he further assumes that the final numbers may extend to $80-90 \%$. These results are based on clinical studies in which participants might be more impaired than in population-based samples. In addition, study participants were younger than in our study.

Long-term studies are prone to selection bias by attrition. Drop-outs from the groups with and without mild cognitive impairment did not differ significantly in age, gender, education or MMSE score at baseline. However, selective attrition may still have occurred with regard to further characteristics. In the calculation of the maximum conversion rate, we used a very conservative approach to minimise any potential problem. This paper focuses on the time-dependency of progression of mild cognitive impairment to dementia. As indicated in Fig. 1, attrition happened gradually and therefore may not obscure the time-dependent pattern.

Studies are needed where people with mild cognitive impairment in different age-groups are followed until their death. Such studies could examine the proportion of people with mild cognitive impairment developing dementia and the proportion dying without a diagnosis of dementia. Age-specific conversion rates of mild cognitive impairment to dementia should be established.

Precise knowledge about age-specific conversion rates of mild cognitive impairment to dementia is essential to make an evidence-based prognosis for people with mild cognitive impairment at a specific age, and to assist in service planning that would include health-economic considerations.

\section{ACKNOWLEDGEMENTS}

This study was supported by the German Bundesministerium für Bildung und Forschung (BMBF), Interdisciplinary Centre for Clinical Research (IZKF) at the University of Leipzig (0IKS9504, Project C7 79934700).

\section{REFERENCES}

American Psychiatric Association (1994) Diagnostic and Statistical Manual of Mental Disorders (4th edn) (DSM-IV). Washington, DC: APA.

Bruscoli, M. \& Lovestone, S. (2004) is $\mathrm{MCl}$ really just early dementia? A systematic review of conversion studies. International Psychogeriatrics, 16, 129-140.

Busse, A., Aurich, C., Zaudig, M., et al (2002) Alters und bildungsspezifische Normierung des kognitiven Tests des SIDAM. Zeitschrift für Gerontologie und Geriatrie, 35, 565-574.

Comijs, H. C., Dik, M. G., Deeg, D. J. H., et al (2004) The course of cognitive decline in older persons: results from the Longitudinal Aging Study Amsterdam.

Dementia and Geriatric Cognitive Disorders, 17, 136-142.

DeCarli, C. (2003) Mild cognitive impairment: prevalence, prognosis, aetiology, and treatment. Lancet Neurology, 2, 15-21.

Folstein, M. F., Folstein, S. E., \& McHugh, P. R. (1975) Mini-Mental State: a practical method for grading the cognitive state of patients for the clinician. Journal of Psychiatric Research, 12, 189-198.

Hughes, C. P., Berg, L., Danziger, W. L., et al (1982) A new clinical scale for the staging of dementia. British Journal Psychiatry, 140, 566-572.

Johansson, B., Zarit, S. H. \& Berg, S. (1992) Changes in cognitive functioning of the oldest old. Journal of Gerontology Series B, 47, P75-80.

Jorm, A. F., Christensen, H., Korten, A. E., et al (1997) Do cognitive complaints either predict future cognitive decline or reflect past cognitive decline? A longitudinal study of an elderly community sample. Psychological Medicine, 27, 91-98.

Palmer, K., Wang, H. X., Backman, L., et al (2002) Differential evolution of cognitive impairment in nondemented older persons: results from the 
Kungsholmen Project. American Journal of Psychiatry, 159 436-442.

Palmer, K., Fratiglioni, L. \& Winblad, B. (2003)

What is mild cognitive impairment? Variations in definitions and evolution of nondemented persons with cognitive impairment. Acta Neurologica Scandinavica Supplementum, 179, 14-20.

Panza, F., D'Introno, A., Colacicco, A.W., et al (2005) Current epidemiology of mild cognitive impairment and other predementia syndromes. American Journal of Geriatric Psychiatry, 13, 633-644.

Petersen, R. C. (2003) Mild Cognitive Impairment Oxford: Oxford University Press.
Petersen, R. C. (2004a) Mild cognitive impairment as a diagnostic entity. Journal of Internal Medicine, 256 183-194.

Petersen, R. C. (2004b) Mild cognitive impairment as a diagnostic entity. Journal of Internal Medicine, 256, 183-194.

Petersen, R. C., Doody, R., Kurz, A., et al (200I) Current concepts in mild cognitive impairment. Archives of Neurology, 58, 1985-1992.

Radloff, L. S. (1977) The CES-D Scale: a self-report depression scale for research in the general population Applied Psychological Measurement, I, 385-40I.

Riedel-Heller, S. G., Busse, A., Aurich, C., et al (200I) Prevalence of dementia according to DSM-III-R and ICD-10: results of the Leipzig Longitudinal Study of the Aged (LEILA75+) Part I. British Journal of Psychiatry, I79, 250-254.

Spitzer, R. L., Williams, J. B. W., Gibbon, M. (1987) Structured Clinical Interview for DSM-III-R (SCID). New York: Biometric Research Department, NYS Psychiatric Institute.

World Health Organization (1992) International Classification of Disorders and Related Health Problems (ICD-10). Geneva: WHO.

Zaudig, M., Mittelhammer, J., Hiller, W., et al (1991) SIDAM: a structured interview for the diagnosis of dementia of the Alzheimer type, multi-infarct dementia and dementias of other aetiology according to ICD-10 and DSM-III-R. Psychological Medicine, 2I, 225-236. 